\title{
EL PRINCIPIO DEL DESARROLLO SOSTENIBLE EN EL CONTEXTO DE LA UNIÓN EUROPEA Y EN EL PLANO INTERNACIONAL
}

The principle of sustainable development in the context of the european union and at the international level

$\underline{\text { Roberto Garetto }}^{126}$

Fecha de recepción: 14 de mayo de 2018

Fecha de aceptación: 23 de mayo de 2018

SUMARIO: 1. El derecho ambiental y el origen del principio del desarrollo sostenible; 2. La evolución del principio de desarrollo sostenible en la Unión Europea y en el contexto internacional; 3. La aplicación del principio del desarrollo sostenible; 4. Referencias Bibliográficas

\footnotetext{
${ }^{126}$ Doctor en Derecho por la Universidad de Camerino, Italia, e investigador becario postdoctoral en la misma Universidad. Vice-director del Instituto Juridico Internacional de Turín, Italia. https://orcid.org/0000-0002-7271-4323 E-mail: roberto.garetto@igito.it
} 


\section{COMO SE CITA ESTE ARTíCULO (APA 6)}

Garetto, Roberto (2018). El Principio del Desarrollo Sostenible en el contexto de la Unión Europea y en el plano internacional. Revista Jurídica Mario Alario D’Filippo, Vol. X №. 20, pág. 173 - 186

RESUMEN: El derecho ambiental ha adquirido una importancia cada vez mayor desde las últimas décadas del siglo XX. El concepto de desarrollo sostenible es fundamental para el derecho ambiental. La evolución de este concepto ha madurado a través de la legislación de la Unión Europea y los pronunciamientos de la ONU. La conciencia sociocultural de la emergencia ambiental ha impuesto al derecho de desarrollar instrumentos adecuados para limitar los daños. La noción de desarrollo sostenible es un requisito previo para las políticas ambientales y las acciones reguladoras. Esta noción coloca en el centro los derechos fundamentales de la persona: la vida, la salud y el bienestar. Las estrategias derivadas del concepto de desarrollo sostenible han favorecido el surgimiento de nuevos principios en el derecho ambiental, como el principio de integración y el principio de «quien contamina paga»; otros están surgiendo: por ejemplo, el principio «quien se beneficia paga».

\section{Palabras Claves}

Derecho ambiental, desarrollo sostenible, políticas ambientales, Unión Europea, ONU, medio ambiente, persona, derechos fundamentales.

ABSTRACT: Environmental law has become increasingly important since the last decades of the twentieth century. The concept of sustainable development is fundamental for environmental law. The evolution of this concept has matured through the legislation of the European Union and the pronouncements of the UN. The sociocultural awareness of the environmental emergency has imposed to the legal thought to develop adequate instruments to limit the damage. The notion of sustainable development is a prerequisite for environmental policies and regulatory actions. This notion puts at the center the person's fundamental rights: life, health and well-being. The strategies derived from the concept of sustainable development favored the emergence of new principles in environmental law, such as the principle of integration and the "polluter pays" principle; others are emerging: for example, the principle «who benefits pays».

\section{Key Words}

Environmental law, sustainable development, environmental policies, European Union, UN, environment, person, fundamental rights. 


\section{El derecho ambiental y el origen del principio del desarrollo sostenible.}

El derecho ambiental se encuentra en una etapa de plena formación; en las últimas décadas se ha desarrollado como una rama autónoma del derecho administrativo tanto a nivel nacional, como a nivel de la UE y en el plano del derecho internacional.

Su creciente importancia va ligada a la trascendencia de los bienes en juego, en defensa del medio ambiente: la calidad de vida, el desarrollo sustentable, la dignidad de la vida humana (Goldenberg, 2004, p.9).

Dentro de la Comunidad, la política medioambiental se ha convertido en un tema del «primer pilar» en 1987 con el Acta Única Europea.

El Tratado de la Unión Europea, firmado en Maastricht el 7 de febrero de 1992, entró en vigor el 1 de noviembre de 1993. El primer acierto del Tratado de Maastricht fue trazar los objetivos de la Unión Europea, disponiendo en su Art. B la promoción de un "progreso económico y social equilibrado y sostenible"127.

Naturalmente, es un requisito previo para la aplicación correcta de cualquier disciplina, incluso de la ley ambiental, que sea bien conocida en sus características fundamentales. Eso, a su vez, lleva a la necesidad de profundizar en la esencia de las instituciones más importantes que se relacionan con el tema conectado con el medio ambiente, entendiendo el valor jurídico que se deriva de ellas, para estar conscientes de la obligación, incluso moral, de respetar y proteger el equilibrio de la naturaleza.

En esta perspectiva, es conveniente tener en cuenta los principios que rigen la legislación ambiental de la Unión Europea. Estos principios deben situarse en el contexto del derecho internacional, captando así la esencia profunda del derecho ambiental y sus vínculos con los equilibrios básicos. A estos equilibrios se refiere precisamente la teoría del desarrollo sostenible afirmada en la Conferencia de Río de 1992, que propuso un perspectiva de superación de la dicotomía economía-ambiente.

Desde este punto de vista, el estudio del derecho ambiental parece ser útil y estimulante, centrándose en la creciente importancia de la gobernanza ambiental. Esto a pesar de que el

${ }^{127}$ Esta adición constituyó un cambio radical, dado que su predecesor hacía solamente mención a «un desarrollo armonioso de las actividades económicas» y una «expansión equilibrada y continuada». Cfr. Reglamento (CEE) núm. 2137/85 del Consejo, de 25 de julio de 1985, relativo a la constitución de una agrupación europea de interés económico (AEIE), accesible en https://eurlex.europa.eu/LexUriServ/LexUriServ.do?uri=CELEX:31985R2137:ES:HTML 
asunto es tan grande y complejo que es imposible considerarlo en su totalidad, por lo que es necesaria una delimitación del campo de estudio. La importancia central del medio ambiente se refleja de hecho en una vasta literatura multidisciplinaria, conectada al derecho en el sentido más amplio, a la ética en la perspectiva jurídica y filosófica, a los fundamentos económicos de las políticas ambientales, a las ciencias exactas.

La dinámica evolutiva y la reconstrucción de la sucesión cronológica de las políticas comunitarias, puede ayudar a comprender la elección de herramientas administrativas identificadas como estratégicas en el sector ambiental.

Desde los años 70 del siglo pasado, bajo la implacable presión de las alarmas provenientes del mundo científico y de los movimientos ecológicos, se celebraron las primeras cumbres internacionales sobre los problemas del medio ambiente y en todos los países industrializados hubo un crecimiento rápido en importancia del derecho ambiental. En la Comunidad Europea, el desarrollo espontáneo de un derecho ambiental subsidiario acompañó al nacimiento y al establecimiento gradual de un sistema generalizado de organismos comunitarios para el control ambiental subsidiario. Esto se basa en la premisa de que muchos problemas de la esfera ecológica van más allá del alcance del estado individual. En el proceso gradual hacia la Unión Europea, se impusieron las políticas ambientales comunes, que posteriormente se tradujeron en el primer pilar, debido a la necesidad obvia de confiar la administración práctica de asuntos ambientales al nivel de gobierno más adecuado para gestionarla.

Las políticas comunitarias en este ámbito son, por lo tanto, bastante recientes y los informes periódicos de la Agencia Europea del Medio Ambiente muestran un panorama medioambiental que aún no permite esperar en mejora sensible y rápida de la situación actual de degradación medioambiental en Europa.

En una correcta reconstrucción de la dinámica del desarrollo del derecho ambiental, debe reconocerse que el crecimiento social y económico surgió antes y que solo más tarde surgieron preocupaciones más conscientes sobre las consecuencias negativas relacionadas con este crecimiento. Sin embargo, la magnitud de estas consecuencias aún no se ha evaluado con precisión. Tampoco se puede decir que ya hayan alcanzado plena efectividad en los Estados miembros aquellos principios reconocidos a nivel internacional, como el principio del desarrollo sostenible, el principio de integración ambiental, el criterio de "quien contamina paga», los principios de precaución, de prevención y corrección del daño, ya incorporados formalmente en los Tratados de la UE y considerados por el derecho internacional como una tendencia hacia un futuro derecho consuetudinario que considera una obligación general del Estado de administrar los recursos naturales de manera razonable. 
Precisamente en el primer principio mencionado, lo del desarrollo sostenible, es deseable elaborar una consideración más ancha, ya que este principio tiene un alcance mayor que los demás, siendo considerado como «el principio de los principios» (Gorosito, 2017, p.115) ${ }^{128}$.

Además, este principio permite poner en relación el medio ambiente con el valor central de la persona, ya que esta debe estar colocada al centro del medio ambiente y en total armonía con él. La centralidad de la persona en el tema del derecho ambiental también permite de poner el medio ambiente en conexión con la protección de los derechos humanos (Mangas, 2018, p. 13) ${ }^{129}$.

\section{La evolución del principio de desarrollo sostenible en la Unión Europea y en el contexto internacional.}

El Tratado constitutivo de la Comunidad Económica Europea del 1957 no atribuía ninguna competencia comunitaria específica en materia de medio ambiente. Esta competencia solo se introducirá en 1987 con el Acta Única Europea (Ragazzo, 2011, p.7).

El principio del desarrollo sostenible se establece tanto en el Tratado de Maastricht del 1992, como en el Tratado de Amsterdam del 1999. Además, este principio ha sido afirmado tanto en el art. $2^{130}$, como en el art. 6 (anteriormente artículo $3 \mathrm{C}$ ) ${ }^{131}$ del Tratado Constitutivo de la Comunidad Europea (Tratado CE).

Sin embargo, de este principio no se ofrece ninguna definición. El Tratado de Maastricht, que convirtió el medio ambiente en un sector oficial de la política de la UE, ya hacía referencia al "crecimiento sostenible», sin definirlo. Ambos conceptos se originan del informe de 1987 de la Comisión Bruntland «Our Common Future» (United Nation, 1987).

El principio del desarrollo sostenible es la piedra angular alrededor de la cual giran las políticas europeas actuales; en este contexto, hablar de «políticas» parece apropiado, ya que, en una perspectiva puramente jurídica, el desarrollo sostenible se configura como un concepto todavía

\footnotetext{
128 «Algunos lo han llamado "el principio de los principios" del Derecho Ambiental hoy: sobre este principio se estructura hoy el paradigma dominante en el campo del Derecho Ambiental, que está inserto en las normas básicas universales, o de jus cogens a nivel internacional y que ha sido constitucionalizado en la mayor parte de los ordenamientos constitucionales del mundo".

129 «El desarrollo sostenible está enraizando como el marco e instrumento de nuevas metas en materia de derechos humanos que sienten las bases de unas condiciones de bienestar que lleven a un nuevo enfoque en materia de derechos humanos".

130 Versión Consolidada del Tratado Constitutivo de la Comunidad Europea, Primera parte - Principios, art. 2: «La Comunidad tendrá por misión promover, mediante el establecimiento de un mercado común y de una unión económica y monetaria y mediante la realización de las políticas o acciones comunes contempladas en los artículos 3 y 4 , un desarrollo armonioso, equilibrado y sostenible de las actividades económicas en el conjunto de la Comunidad, un alto nivel de empleo y de protección social, la igualdad entre el hombre y la mujer, un crecimiento sostenible y no inflacionista, un alto grado de competitividad y de convergencia de los resultados económicos, un alto nivel de protección y de mejora de la calidad del medio ambiente, la elevación del nivel y de la calidad de vida, la cohesión económica y social y la solidaridad entre los Estados miembros».

131 Versión Consolidada del Tratado Constitutivo de la Comunidad Europea, Primera parte - Principios, art. 6: «Las exigencias de la protección del medio ambiente deberán integrarse en la definición y en la realización de las políticas y acciones de la Comunidad a que se refiere el artículo 3 , en particular con objeto de fomentar un desarrollo sostenible».
} 
vago (Krämer, 2002, p.71). Queda claro que desde un punto de vista científico, socioeconómico y cultural, es un principio de importancia capital. Jurídicamente, su entrada en el Tratado constituye un punto de inflexión histórico, teniendo en cuenta los principios meramente económicos en los que se basó previamente la Comunidad. Sin embargo, se trata de una introducción elaborada a través de una reflexión política gradual.

El objetivo de una situación de equilibrio del desarrollo con el medio ambiente implica tanto la protección del medio ambiente, como el desarrollo económico. La herramienta para su implementación en la Unión es la integración de la política medioambiental en todas las demás políticas europeas. La conexión lógica entre los dos conceptos es que la implementación del desarrollo sostenible implica necesariamente la integración de la protección del medio ambiente en todas las demás políticas europeas. El proceso de adquisición de una estrategia europea de integración ambiental se origina a partir de consideraciones básicas e intuitivas: las actividades humanas afectan el medio ambiente; el consumo de energía es una fuente de contaminación y la urbanización daña los ecosistemas. Para evitar un daño ambiental irreversible, es necesario que todas las políticas tengan en cuenta el medio ambiente: esto significa implementar el desarrollo sostenible.

Con el tiempo, los requisitos de protección ambiental han adquirido una importancia creciente en comparación con otras políticas comunitarias, hasta el establecimiento formal del Acta Única Europea. El Tratado de la Unión Europea se ocupa de cuestiones medioambientales en los artículos 174 (antiguo artículo 130 R), 175 (antiguo artículo 130 S) y 176 (antiguo artículo 130 T) del Tratado CE. Este mismo tratado afirma el principio de integración de la protección del medio ambiente en las políticas comunitarias en el art. 6; en este artículo, el principio de integración se establece como una base para la acción comunitaria, un instrumento operativo cuyo objetivo final sigue siendo implementar el desarrollo sostenible, es decir, un desarrollo conforme a las necesidades del presente, pero consciente de los derechos de las generaciones futuras.

Intentando aplicar el principio de integración establecido en el Tratado de Maastricht, en 1998 la Comunidad lanzó una estrategia, el «Proceso de Cardiff» ${ }^{132}$, con el objetivo de lograr resultados concretos en los sectores más perjudiciales para el medio ambiente. La estrategia conectada al proceso de Cardiff originó una serie de acciones comunitarias con el objetivo de integrar la protección del medio ambiente en las diversas políticas económicas ${ }^{133}$.

132 Consejo Europeo de Cardiff: Ios días 15 y 16 de junio de 1998 se reunieron en Cardiff los jefes de Estado y de gobierno de la UE. Para una valoración de este proceso cfr. el trabajo colectivo de M. FERGUSON, C. COFFEY, D. WILKINSON y D. BALDOCK, The effectiveness of EU Council Integration Strategies and Options for carrying forward the "Cardiff" Process, IEEP, Brussels, 2001.

133 Desde la Cumbre de Cardiff se fueron presentando por la Comisión propuestas sobre integración ambiental de determinadas políticas: energía (octubre de 1998), agricultura (enerode 1999), mercado interior, industria y desarrollo (junio de 1999), pesca (en julio de 1999) y medio urbano (noviembre de 1999). Sobre este tema cfr. F.J. SANZ LARRUGA, "La integración europea y el principio comunitario de integración ambiental en el ordenamiento jurídico español”, Rev. secr. Trib. perm. Revis., 6, núm. 12, 2018, pp. 262 s. 
La Unión Europea fue uno de los protagonistas de la Cumbre Mundial sobre el Desarrollo Sostenible celebrada en Johannesburgo en 2002, subscribiendo al final sus resultados (Declaración de Johannesburgo sobre Desarrollo Sostenible, aprobada en la 17 sesión plenaria, celebrada el 4 de septiembre, 2002) y garantizando su implementación en una comunicación adoptada al año siguiente, el Consejo de la Unión de Medio Ambiente de octubre de 2002.

La «Declaración de Johannesburgo sobre el Desarrollo Sostenible. Desde nuestro origen hasta el futuro» (ONU, s.f.), firmada por los Estados participantes en la Cumbre Mundial sobre el Desarrollo Sostenible celebrada en Johannesburgo entre el 26 de agosto y el 4 de septiembre de 2002, reafirma el principio del desarrollo sostenible a nivel internacional y las interdependencias entre los problemas ambientales y sociales.

El documento afirma la importancia de reducir «la línea profunda que divide la sociedad humana entre los ricos y pobres y la siempre creciente brecha entre los mundos desarrollados y en desarrollo» (Declaración de Johannesburgo sobre el Desarrollo Sostenible, 2-4 de septiembre 2002, n.12) a fin de garantizar la estabilidad y la prosperidad global. Para alcanzar el objetivo de la sostenibilidad, el compromiso solemne de todos los países de actuar juntos, unidos por la determinación común de salvar el planeta y promover el desarrollo humano, en «un proceso inclusivo» (Declaración de Johannesburgo sobre el Desarrollo Sostenible, 2-4 de septiembre 2002, n. 34) ${ }^{134}$ que tienda a asegurar que se logre la esperanza colectiva para el desarrollo sostenible de las poblaciones del mundo y de las generaciones futuras.

Durante la cumbre de Johannesburgo con la declaración política adjunta sobre el desarrollo sostenible, se adoptó un Plan de Acción. Este plan define de manera concreta los objetivos funcionales para lograr el desarrollo sostenible a través de una especie de hoja de ruta. Entre estos, los más significativos, en resumen, son: el acceso al agua y estrés hídrico; uno de los objetivos era reducir para el año 2015 la población que no disponía de saneamiento adecuado para las aguas residuales. La energía; las reservas de combustibles fósiles estarán en riesgo de agotamiento en las próximas décadas, y el petróleo será prohibitivo para los países del sur y las energías renovables y sus usos siguen estando fuera del alcance de muchos países emergentes. La producción agrícola, que a través de la agricultura intensiva suscita algunos revuelos en la comunidad científica y entre los consumidores; otros sistemas de producción agrícola, tal como la agricultura biológica, pero siguen fuera del alcance de los países del Sur que luchan para alimentarse a sí mismos. La biodiversidad de las especies animales; bajo el efecto de la presión demográfica numerosas especies de plantas y animales desaparecen bajo la influencia de la

134 «Estamos de acuerdo que este debe ser un proceso inclusivo, involucrando a todos los grupos principales y a los gobiernos que participaron en la histórica Cumbre de Johannesburgo». 
deforestación o de la destrucción de su medio ambiente. La salud; las poblaciones del Sur siguen careciendo de acceso a las farmacopeas de los países desarrollados. Y, finalmente, el clima, en relación con el cual se reafirmaron los compromisos contraídos en la Convención de Río sobre el cambio climático (Report of the United Nations Conference on Environment and Development, 1992), y se solicitó a los países que aún no lo hayan hecho que ratifiquen el Protocolo de Kyoto.

El Consejo Europeo confirmó en diciembre de 2009 que «en el marco del Tratado de Lisboa el desarrollo sostenible sigue siendo un objetivo el desarrollo sostenible sigue siendo un objetivo fundamental de la Unión Europea» (Consejo Europeo 10 y 11 de Diciembre, 2009), el desarrollo sostenible es una preocupación global; por el momento, sus principios deben ser implementados más ampliamente en la cooperación internacional y la política de desarrollo.

La revisión de la estrategia para un desarrollo sostenible por parte de la Comisión Europea (EDS UE) en 2009 destacó la persistencia de algunas tendencias no sostenibles y la necesidad de un mayor compromiso con ellas. Sin embargo, también señaló el progreso realizado por la Unión Europea en la integración del desarrollo sostenible en muchas de sus políticas (incluido el comercio y el desarrollo) y destacó el progreso en el cambio climático y la promoción de una economía baja en carbono ${ }^{135}$.

La Comunicación de la Comisión al Consejo y al Parlamento Europeo «Río+20: hacia una economía verde i una mejor gobernanza» ${ }^{136}$, de 2011, también incluye una sección sobre desarrollo sostenible. La comisión se refiere a la Estrategia Europa 2020 como una herramienta valiosa para lograr el desarrollo sostenible en la Unión Europea.

Como se destaca en el informe de la Presidencia sobre la revisión de 2009 de la Estrategia de Desarrollo Sostenible de la Unión, la estrategia seguirá proporcionando una visión a largo plazo y constituirá el marco político general para todas las políticas y estrategias de la Unión. Varias tendencias no sostenibles requieren una acción urgente: se necesitan esfuerzos adicionales significativos para frenar y adaptarse al cambio climático, disminuir el alto consumo de energía

\footnotetext{
135 Cfr. Comunicación de la Comisión al Parlamento Europeo, al Consejo, al Comité Económico y Social Europeo y al Comité de las Regiones - Incorporación del desarrollo sostenible en las políticas de la UE : informe de 2009 sobre la estrategia de la Unión Europea para el desarrollo sostenible, 24.7.2009, $\operatorname{COM(2009)~} 400$ final. En particular este pasaje: «En los últimos años, la Unión Europea ha integrado el objetivo de desarrollo sostenible (DS) en una gama amplia de políticas. La UE se encuentra en la vanguardia, a nivel internacional, de la lucha contra el cambio climático y está comprometida a crear una economía con unas bajas emisiones de carbono, basada en el conocimiento y con un uso eficiente de los recursos. Al mismo tiempo, persisten ciertas tendencias inviables en varios ámbitos, a pesar de toda una serie de avances políticos positivos. La demanda de recursos naturales ha crecido rápidamente y supera los recursos naturales disponibles del planeta a largo plazo. Se está perdiendo biodiversidad en términos globales y los principales ecosistemas están sufriendo una presión creciente. El consumo de energía en los transportes continúa creciendo. Persiste la pobreza a nivel mundial; $y$ deben realizarse grandes esfuerzos para alcanzar los Objetivos de Desarrollo del Milenio". Véase el texto completo en: https://eur-lex.europa.eu/legal-content/ES/TXT/?uri=celex\%3A52009DC0400

136 Comunicación de la Comisión al Parlamento Europeo, al Consejo, el Comité Económico y Social Europeo e al Comité de las Regiones, "Río+20: hacia una economía verde i una mejor gobernanza", 20.6.2011, COM(2011) 363 final. Véase el texto completo en: https://eur-lex.europa.eu/LexUriServ/LexUriServ.douri=COM:2011:0363:FIN:EN: PDF
} 
en el sector del transporte y revertir la pérdida actual de biodiversidad y recursos naturales. El cambio hacia una economía segura y sostenible con bajas emisiones de carbono requerirá un mayor enfoque en el futuro. Las acciones prioritarias deberían especificarse más claramente en futuras revisiones. La gobernanza, incluidos los mecanismos de aplicación, supervisión y seguimiento, debe reforzarse, por ejemplo, mediante vínculos más claros con la futura estrategia UE 2020 y otras estrategias transversales. En la UE, una cuestión clave es integrar el pensamiento sobre el desarrollo sostenible en varias partes de la Estrategia Europa 2020.

La Estrategia, que fue adoptada en 2010, contribuyó a sacar a Europa de la crisis y sentar las bases para un futuro más sostenible basado en un crecimiento inteligente, sostenible e integrador ${ }^{137}$. Las áreas clave para la integración son el Fondo Europeo para la Inversión Estratégica (FEIE) (Pérez \& Pizzarro, 2012, p.230), a través de la unión energética y la política climática, pero en particular el trabajo sobre la economía circular (Frerot, 2014, p.5).

Este enfoque horizontal está en consonancia con el «principio de integración» (Fernández, 2003, p.78) $)^{138}$ del Tratado Constitutivo de la Comunidad Europea, tal como se establece en el artículo 11, y hasta ahora ha demostrado su utilidad. El desarrollo sostenible es una preocupación global; por lo tanto, sus principios deben ser implementados más ampliamente en la cooperación internacional y la política de desarrollo.

En el ámbito del derecho internacional, durante los últimos años muchas son las iniciativas. Recordamos en particular el antes mencionado (en reacción a un documento de la UE) «Rio+20», que es el nombre abreviado de la Conferencia de las Naciones Unidas sobre el Desarrollo Sostenible, que tuvo lugar en Río de Janeiro del 20 al 22 de junio de 2012 (Griggs, Stafford-Smith, Gaffney, Rockström, Öhman, Shyamsundar, Steffen, Glaser \& Kanie, Noble,2013, p.305), veinte años después de la histórica Cumbre de la Tierra en Río en 1992.

En la Conferencia Río +20 , los líderes mundiales se unieron para dar forma a la manera en que puede reducir la pobreza, fomentar la equidad social y garantizar la protección del medio ambiente en un planeta cada vez más poblado.

\footnotetext{
137 «la crisis económica mundial de los últimos años ha obligado a las autoridades y responsables públicos a reflexionar sobre cómo encarar sus efectos, manteniendo objetivos y elementos que se consideran prioritarios y que forman parte del acervo en el seno de la Unión Europea. La Estrategia Europa 2020 (Comisión Europea, 2010a) es una respuesta al nuevo contexto y a los retos que se derivan de las convulsiones sociales y económicas recientes y de sus efectos negativos, pero también al mantenimiento de unos objetivos globales para el conjunto de la Unión Europea, como son el de un desarrollo sostenible y equilibrado desde el punto de vista social, económico y territorial». O. GARCÍA LUQUE, Ú. FAURA MARTínEZ y M. LAFUENTE LECHUGA, "Objetivo Europa 2020. La reducción de la pobreza y la exclusión social en España", Papers, núm. 101/4, 2016, p. 505: «Dicha estrategia constituye el nuevo marco comunitario de acción para el periodo 2010-2020, en respuesta a la crisis económica, y se enuncia en cinco objetivos tendentes a lograr un crecimiento económico inteligente, sostenible e integrador».

138 «El principio de integración, entendido como la necesidad de incorporar el componente medioambiental en todas aquellas políticas que tienen efectos negativos sobre el entorno con el objetivo de mejorar los resultados de la política de medio ambiente (o de reducir su déficit de implementación)».
} 
Las conversaciones oficiales se centraron en dos temas principales: cómo construir una economía ecológica para lograr el desarrollo sostenible y sacar a la gente de la pobreza, y cómo mejorar la coordinación internacional para el desarrollo sostenible.

El 19 de junio de 2012 las 193 delegaciones que participan en la Conferencia Río+20 alcanzaron un acuerdo de mínimos sobre el borrador de conclusiones titulado «El futuro que queremos» ${ }^{139}$.

\section{La aplicación del principio del desarrollo sostenible.}

La modificación de las condiciones ambientales, desde un punto de vista socioeconómico, fue llevada a cabo por el hombre para mejorar la calidad de su existencia. Como se ha observado, «el uso ilimitado que ha realizado el hombre de los bienes y servicios ambientales ha dado lugar a profundas modificaciones dentro de su entorno, las cuales repercuten en forma última, sobre su calidad de vida. El reconocimiento de estas modificaciones dio pie a la creación de medidas jurídicas encaminadas a la protección y uso racional del ambiente» (Vázquez \& Silva, s.f, p.11).

Durante las últimas décadas del siglo $X X$, sin embargo, se ha entendido que es un límite al aumento del progreso si el daño que produce al medio ambiente es mayor que aquellos que el medio ambiente puede soportar sin convertirse en peligroso para los humanos. Sin embargo, las políticas ambientales hasta ahora no han podido desviar la creciente demanda de "progreso", que ha crecido significativamente en los últimos años; esto demuestra que las alarmas ambientales en sí mismas no han tenido un verdadero efecto disuasorio en la opinión pública, y mucho menos han contribuido a incrementar su responsabilidad ambiental.

Un concepto de limitación del desarrollo en la perspectiva de la amenaza al equilibrio global necesario para la vida humana ya se había introducido en los años 70 del siglo pasado, cuando científicos de todo el mundo se reunieron en Estocolmo para la primera conferencia sobre el medio ambiente y estuvieron de acuerdo en que este tipo de desarrollo estaba dañando el medio ambiente irreversiblemente. Era lógico y consecuente hablar entonces de «límites», pero el límite referido era precisamente lo del crecimiento económico, requisito previo para el desarrollo occidental, es decir: el bienestar. En ese momento, el concepto de limitación al desarrollo era sinónimo de inversión del camino hacia el bienestar, de acuerdo con el principio de que si la explotación de los recursos naturales es lo que sostiene el crecimiento económico, entonces el desarrollo económico y la protección del medioambiente eran incompatibles. Poner un límite, de acuerdo con esta concepción, todavía implicaría una desaceleración en el crecimiento, si el propósito fuera permitir la regeneración de la naturaleza. La idea que subyace al concepto de limitación es que el bienestar, como bienestar artificial, crece directamente proporcional a la degradación ambiental.

139 Accesible en https://rio20.un.org/sites/rio20.un.org/files/a-conf.216-l-1 spanish.pdf.pdf 
La consecuencia del desarrollo económico incontrolado fue una degradación más alta que el nivel de equilibrio ambiental. Esto determinó la necesidad de poner un límite cuantitativo al desarrollo. Mientras tanto se iba afirmando la conciencia, corroborada por las ciencias exactas, de que el medio ambiente no tiene una capacidad infinita para regenerar sus recursos en un tiempo limitado y que, por lo tanto, existe un nivel de equilibrio ambiental no superable.

Si toleramos que la actividad humana altere el equilibrio ecológico más allá de un cierto límite, el tiempo para la autorregeneración del medio ambiente podría ser tan largo no solo para afectar la calidad de vida de las generaciones futuras, sino también para crear, a largo plazo. Serios problemas de naturaleza económica.

En la actualidad, las perspectivas han cambiado radicalmente (Cafferatta, 2004, p18), pero la tendencia a considerar el deterioro del medio ambiente como un aspecto inevitable del desarrollo ha prevalecido durante gran parte del siglo XX. El tema del desarrollo sostenible se vuelve actual solo desde los años ochenta del siglo pasado. En Octubre de 1984 se reunió por primera vez la Comisión Mundial sobre Medio Ambiente y Desarrollo (World Commission on Environment and Development). La Comisión partió de la convicción de que es posible para la humanidad construir un futuro más próspero, más justo y más seguro. Con ese enfoque optimista publicó en abril de 1987 su informe denominado "Nuestro Futuro Común» (Our Common Future), o Informe Brundtland, que ya hemos mencionado. La Comisión planteó que la humanidad tiene la capacidad para lograr un «desarrollo sostenible», al que definió como aquel que garantiza las necesidades del presente sin comprometer las posibilidades de las generaciones futuras para satisfacer sus propias necesidades. La Comisión afirmó la necesidad de encontrar un equilibrio entre el desarrollo socioeconómico y la protección del medio ambiente.

A partir de ese momento el concepto de desarrollo sostenible comenzó a afirmarse.

La Declaración de Río sobre el Medio Ambiente y el Desarrollo, de 7 de mayo de 1992, surgida de la Conferencia de las Naciones Unidas, reunida en Río de Janeiro del 3 al 14 de junio de 1992, acogió esta filosofía, superando el prejuicio de que había una incompatibilidad lógica absoluta entre la idea de bienestar hasta entonces aceptada y el concepto de limitación a la explotación ambiental. La noción de desarrollo sostenible tiene una matriz económica, pero su concepto amplio generalmente se considera un enfoque válido para la formulación de políticas, teniendo en cuenta las perspectivas a largo plazo y la interacción entre las diferentes posibilidades de desarrollo y las acciones políticas; de hecho, la tarea específica de la política es determinar un equilibrio unitario, no sectorial, en la relación entre economía y medio ambiente. El desarrollo sostenible, originalmente conceptualizado en la esfera económica, ha pasado por el campo 
político, ya que se considera un enfoque general eficaz para la definición de todas las políticas, teniendo en cuenta las perspectivas a largo plazo y la interacción entre las diferentes opciones de desarrollo y de acciones políticas.

La sostenibilidad del desarrollo es una idea del equilibrio económico ambiental. Para ser sostenible, el desarrollo no debe causar daños más allá de un cierto límite de equilibrio y los responsables de las actividades perjudiciales deben tener en cuenta el costo de las medidas de protección y restauración. Aquellos que tienen la responsabilidad política de impulsar el desarrollo deben hacer una evaluación exacta de los costos ambientales, ya que, si el crecimiento del progreso no debe parar, es necesario estimar los costos la prevención o reparación del daño producido. Estos son costos que, por lo tanto, deben imputarse a quienes explotan los recursos naturales más allá del interés colectivo; de ahí la idea de implementar el principio de «quien contamina paga». La evaluación de costos podría ser parte de la planificación financiera de la actividad comercial ${ }^{140}$, pero actualmente no se ha establecido un «mercado ambiental» y muchos de los recursos naturales se usan sin ser incluidos en los costos de producción.

Por lo tanto, los costos ambientales todavía no pueden verse como un elemento específico del presupuesto de una empresa, ya que aún no se ha cuantificado el precio por el uso indebido de los recursos naturales. En los casos más graves, hay enormes costos de recuperación y en los últimos treinta años, dentro de la Unión Europea, es tema controvertido si deben ser pagados completamente por los responsables, bajo qué condiciones y con qué modalidades. Más recientemente se está afirmando el principio "quien se beneficia paga», según el cual aquellos que reciben o esperan recibir beneficios por actividades de control de la contaminación o acciones de conservación deberían pagar por dicho beneficio (Borregaard y T. Bradley, 1999, p.57).

Este debate confirma la centralidad del tema del desarrollo sostenible, que en las últimas décadas se ha establecido como imprescindible en las políticas comunitarias e internacionales. La conciencia de una emergencia ambiental en un nivel sociocultural ha conducido inevitablemente a intervenciones en el campo del derecho, así como de la economía. Se necesita hacer mucho más, pero el camino trazado es el correcto.

\footnotetext{
140 «la necesidad de homogeneizar la definición sobre inversiones ambientales, estimular una discusión sobre los pasivos ambientales y sus implicancias en términos económicos y de responsabilidad legal, generar debate en torno al aporte de los análisis económicos, y promover más investigación sobre la valorización económica». En una perspectiva económica y de responsabilidad y legal, sería posible, por ejemplo, contener el impacto de los costos para el riesgo ambiental a través del uso de compañías de seguros.
} 


\section{REFERENCIAS BIBLIOGRAFICAS}

FRÉROT, (2004) Economía circular y eficacia en el uso de los recursos: un motor de crecimiento económico para Europa, Fundación Robert Schuman / Cuestión de Europa, núm. 331.

AGUILAR, (2003) El principio de integración medioambiental dentro de la Unión Europea: la imbricación entre integración y desarrollo sostenible, Papers, núm. 71.

BORREGAARD y T. Bradley, Impactos ambientales del comercio: Análisis de tres sectores exportadores chilenos, Ambiente y Desarrollo, vol. 15, núm. 4, 1999.

CAFFERATTA, (2004) Introducción al derecho ambiental, Introducción al derecho ambiental, Instituto Nacional de Ecología (INE-SEMARNAT), Ciudad de México.

ESPARCIA Y Escribano, (2012) La dimensión territorial en la programación comunitaria y el nuevo marco de políticas públicas: desarrollo rural territorial, reforma de la PAC y nuevo LEADER, Anales de Geografía, vol. 32, núm. 2.

FERGUSON, Coffey, Wilkinson \& Baldock, (2001). The effectiveness of EU Council Integration Strategies and Options for carrying forward the "Cardiff" Process, IEEP, Brussels.

GARCÍA, (2018) Del principio quien contamina paga al principio quien se beneficia paga: nuevos instrumentos económicos en materia ambiental, Veredas do Direito, vol. 15, núm. 31.

GARCÍA, Faura \& Lafuenta, (2016) Objetivo Europa 2020. La reducción de la pobreza y la exclusión social en España, Papers, núm. 101/4.

GOLDENBERG, Prólogo, en CAFFERATTA, Introducción al derecho ambiental, cit.

GOROSITO, (2017) "Los principios en el Derecho Ambiental”, Revista de Derecho (UCUDAL), 2da época, 13, núm. 16.

GRIGGS, Stafford-Smith, Gaffney, Rockström, öhman, Shyamsundar, Steffen, Glaser, Kanie \& Noble, (2013) "Policy: Sustainable development goals for people and planet", Nature, vol. 495, 21 de Marzo.

KRÄMER, (2002) Manuale di diritto comunitario per l'ambiente, Giuffrè, Milano. 
MANGAS, (2018) "Unión Europea derechos humanos y desarrollo sostenible", en C.R. FERNÁNDEZ LIESA y C.M. DÍAZ BARRADO (a cargo de), "Objetivos de desarrollo sostenible y derechos humanos: paz, justicia e instituciones sólidas / derechos humanos y empresas", Instituto de Estudios Internacionales y Europeos Francisco de Vitoria de la Universidad Carlos III de Madrid, núm. 9.

RAGAZZO, (2011) Le politiche sull'energia e le fonti rinnovabili, Giappichelli, Torino.

VÁZQUEZ y Silva, Presentación, en N.A. CAFFERATTA, Introducción al derecho ambiental, cit. 